Research Paper

\title{
Effects of Active Mastication on Chronic Stress-Induced Bone Loss in Mice
}

\author{
Kagaku Azuma1, ${ }^{1,}$, Manabu Furuzawa², Shu Fujiwara², Kumiko Yamada³, Kin-ya Kubo ${ }^{4}$ \\ 1. Department of Anatomy, School of Medicine, University of Occupational and Environmental Health, Fukuoka, 807-8555, Japan \\ 2. Department of Prosthodontics, Asahi University School of Dentistry, Mizuho, 501-0296, Japan \\ 3. Department of Anatomy and Physiology, Faculty of Domestic economy, Nagoya Women's University, Nagoya, 467-8610, Japan \\ 4. Seijoh University Graduate School of Health Care Studies, Tokai, 478-8588, Japan
}

$\triangle$ Corresponding author: Kagaku Azuma, Department of Anatomy, School of Medicine, University of Occupational and Environmental Health, 1-1 Iseigaoka, Yahatanishi-ku, Kitakyusyu, Fukuoka, 807-8555, Japan. TEL: +81 93691 7418; FAX: +81 93691 8544; E-mail:kazuma@med.uoeh-u.ac.jp

(C) 2015 Ivyspring International Publisher. Reproduction is permitted for personal, noncommercial use, provided that the article is in whole, unmodified, and properly cited. See http://ivyspring.com/terms for terms and conditions.

Received: 2015.07.20; Accepted: 2015.10.07; Published: 2015.11.04

\begin{abstract}
Chronic psychologic stress increases corticosterone levels, which decreases bone density. Active mastication or chewing attenuates stress-induced increases in corticosterone. We evaluated whether active mastication attenuates chronic stress-induced bone loss in mice. Male C57BL/6 (B6) mice were randomly divided into control, stress, and stress/chewing groups. Stress was induced by placing mice in a ventilated restraint tube $(60 \mathrm{~min}, 2 \times /$ day, 4 weeks). The stress/chewing group was given a wooden stick to chew during the experimental period. Quantitative micro-computed tomography, histologic analysis, and biochemical markers were used to evaluate the bone response. The stress/chewing group exhibited significantly attenuated stress-induced increases in serum corticosterone levels, suppressed bone formation, enhanced bone resorption, and decreased trabecular bone mass in the vertebrae and distal femurs, compared with mice in the stress group. Active mastication during exposure to chronic stress alleviated chronic stress-induced bone density loss in B6 mice. Active mastication during chronic psychologic stress may thus be an effective strategy to prevent and/or treat chronic stress-related osteopenia.
\end{abstract}

Key words: Chewing, Corticosterone, Osteoporosis, Psychologic stress, X-ray microtomography

\section{Introduction}

Chronic psychologic stress is a major health concern worldwide as it affects multiple physiologic systems. Stress is a risk factor for several different diseases, including inflammatory disease, hypertension, cardiovascular disease, obesity, diabetes, atherosclerosis, cancer, as well as neurodegenerative diseases [1-3]. Exposure to psychologic stressors activates the central hypothalamic-pituitary-adrenocortical (HPA) axis [4], leading to the synthesis and release of corticotropin-releasing hormone, which increases the secretion of adrenocorticotropic hormone. Adrenocorticotropic hormone stimulates the synthesis and release of glucocorticoids into the systemic circulation.

Osteoporosis is an increasingly common skeletal disease that increases the risk of fracture, character- ized by low bone mass and bone structure deterioration [5]. Several factors are implicated in the development of osteoporosis, such as menopause, Caucasian or Asian race, thin skeletal frame, physical inactivity, smoking, alcohol use, inadequate calcium and vitamin D intake, corticosteroid treatment, and certain medical conditions [5-7]. Extensive evidence from both animal and human studies indicates an association between chronic psychologic stress and osteoporosis [8-10], suggesting that stress may aggravate or trigger bone density loss [11]. High glucocorticoid levels inhibit osteoblast differentiation and bone formation, and induce osteoblast apoptosis, thereby suppressing bone growth $[12,13]$, while at the same time extending the lifespan of mature osteoclasts, leading to an imbalance in the osteoblast-osteoclast 
ratio and eventual bone density loss.

Chewing is important not only for food intake, but also for mental, physical, and physiologic functions of the body [14-17]. The act of chewing defends against various stressors by suppressing the neuroendocrine stress response in the HPA axis [18-22]. The effect of chewing to suppress activation of the HPA axis suggests that chewing during exposure to stress could attenuate chronic stress-induced bone loss. We recently reported that senescence-accelerated mouse strain 8 (SAMP8) exposed to chronic stress exhibited bone loss. We therefore hypothesized that allowing mice to chew on a wooden stick during exposure to chronic stress could ameliorate the stress-induced bone loss [23].

Bone mass and bone architecture appear to be strain-specific. C57BL/6J (B6) mice have a relatively lower bone mass and higher endosteal bone resorption rate [24-26]. Furthermore, genetically different inbred murine strains exhibit distinct behavioral responses to chronic stress [27]. The most widely used inbred murine strain, B6 mice, show reduced latency to feed in a novelty-suppressed feeding test and increased interaction times in the social interaction test, suggesting decreased anxiety in a stressful environment [27]. In the present study, we investigated static and dynamic bone histomorphometry, and bone turnover to evaluate whether chewing under chronic stress conditions prevents stress-induced bone loss in B6 mice.

\section{Materials and Methods}

\section{Animals and experimental procedures}

Male B6 mice (12 months of age) were purchased from Japan SLC Inc. (Shizuoka, Japan) and housed in the Division of Animal Experiments, Asahi University School of Dentistry under temperature- and humidity-controlled conditions $\left(23 \pm 1^{\circ} \mathrm{C}, 55 \pm 25 \%\right)$ and a $12: 12$ h light/dark cycle (light period, 6:00-18:00; dark period, 18:00-6:00). A standard rodent pellet chow (CE-2, CLEA Japan, Inc., Tokyo, Japan) containing $1.03 \%$ calcium and $0.97 \%$ phosphorus was provided to the mice ad libitum. All experiments were performed according to the guidelines for the care and use of laboratory animals of Asahi University. The mice were randomly distributed into control, stress, and stress/chewing groups ( $\mathrm{n}=10 /$ group). Mice were exposed to stress for $60 \mathrm{~min}$, twice a day, for 4 weeks by placing them in a ventilated plastic restraint tube (inner diameter: $3.5 \mathrm{~cm}$ ), in which they could move back and forth but not turn around, as previously reported $[18,23]$. While in the restraint tube, the mice were also subjected daily to either water immersion, physical shaking, or flashing lights. To prevent ha- bituation and provide an unpredictable aspect to the stressors, all stressors and/or sequences were administered at different times of the day. Mice in the stress/chewing group were allowed to chew on a wooden stick (diameter: $2 \mathrm{~mm}$ ), made from virgin white birch tree, for $60 \mathrm{~min}$, twice a day during the experimental period $[18,23]$. The wooden sticks were examined for signs of chewing at the end of the experimental period and all of them showed signs of chewing.

\section{Serum biochemical parameters}

At the end of the experiments, the mice were given an overdose of sodium pentobarbital by intraperitoneal injection. Blood was obtained from the axillary artery and serum corticosterone levels were measured using a quantitative competitive ELISA kit (AssayPro, St. Charles, MO, USA). Serum levels of osteocalcin, an osteoblast bone formation marker, were determined using a mouse osteocalcin enzyme-linked immunoassay kit (Biomedical Technologies, Stoughton, MA, USA). Serum levels of $\mathrm{N}$-telopeptide of type I collagen (NTx), an osteoclast bone resorption marker, were measured using an enzyme-linked immunosorbent assay kit (TECOmedical, Sissach, Switzerland).

\section{Micro-CT imaging and three-dimensional as- sessment of trabecular bone}

Mouse femurs and fourth lumbar vertebrae (L4) were dissected by removing adherent soft tissues and then maintained in sealed plastic bags at $-20^{\circ} \mathrm{C}$. Trabecular microstructure was analyzed using a cone-beam X-ray micro-CT system (MCTCB100MF, Hitachi Medical Corporation, Kashiwa, Japan) as described previously [23, 28-30]. The L4 vertebral body and distal femur were scanned at $10-\mu \mathrm{m}$ resolution with a tube voltage of $50 \mathrm{kV}$, a tube current of $0.1 \mathrm{~mA}$, and a voxel size of $10 \mu \mathrm{m}$. After scanning, the micro-CT image data was transferred to a workstation. The region of interest was defined using 100 slices at approximately $0.5-2.0 \mathrm{~mm}$ away from the growth plate of the distal femur. The trabecular bone area of the vertebral body was outlined for each micro-CT slice, excluding the cranial and caudal endplate regions. Within these regions, trabecular bone was separated from cortical bone with boundaries defined by the endocortical bone surfaces. The structural indices were calculated using three-dimensional (3D) trabecular bone analysis software TRI/3D-BON (Ratoc System Engineering Co. Ltd., Tokyo, Japan), which constructs 3D models from serial tomographic datasets for visualization and morphometric analysis, as described previously $[23,30]$. 


\section{Histomorphometric measurements}

Mice were injected intraperitoneally with calcein (10 mg/kg body weight) 8 and 2 days before dissection of the femurs and vertebrae after micro-CT scanning, and embedded in methyl methacrylate. The vertebral body was cut at $150-\mu \mathrm{m}$ thickness using a low speed diamond saw (Isomet, Buehler, Lake Bluff, IL, USA), and hand ground to a thickness of $20 \mu \mathrm{m}$ for dynamic bone histomorphometric analyses. The bone mineral surface, mineral apposition rate, and bone formation rate per bone surface (BFR/BS) were measured. For paraffin block preparations, bone specimens were fixed in freshly prepared $4 \%$ paraformaldehyde solution, decalcified in 5\% EDTA solution, embedded in paraffin, and sectioned using standard histologic protocols, as described previously $[23,31]$. Sections $(5 \mu \mathrm{m})$ were prepared for tartrate-resistant acid phosphatase staining to evaluate osteoclast number per bone surface (N.Oc/BS) and osteoclast surface (Oc.S/BS). All bone histomorphometric measurements were performed using Adobe Photoshop, as previously described [32].

\section{Statistical analysis}

All data are reported as mean \pm SD. The statistical analysis was performed using SPSS version 14 (Chicago, IL, USA). A one-way analysis of variance was used to compare trabecular and cortical microstructural parameters in the control, stress, and stress/chewing groups. If the $\mathrm{F}$ test indicated significance, Fisher's protected least significant differences test was used for post hoc multiple comparisons. $\mathrm{P}$ value of less than 0.05 was considered to indicate statistical significance.

\section{Results}

Serum corticosterone levels were significantly higher in the stress group compared with the control group. Chewing on a wooden stick while under chronic stress inhibited the increase in the serum corticosterone levels (Fig. 1). Serum osteocalcin levels were significantly lower and serum NTx levels were significantly higher in the stress group compared with the stress/chewing group (Fig. 1).

The microstructural parameters of the vertebral trabecular bone are shown in Fig. 2. The trabecular bone volume fraction $(\mathrm{BV} / \mathrm{TV})$, trabecular number (Tb.N), and trabecular thickness (Tb.Th) were significantly lower in the stress group compared with the control group. Trabecular separation (Tb.Sp) was significantly higher in the stress group (Fig. 2). BV/TV and Tb.N were significantly higher, and Tb.Sp was significantly lower in the stress/chewing group than in the stress group. Typical 3D reconstructed micro-CT images of the L4 vertebral body are shown in
Fig. 3. Images of samples with a BV/TV value closest to the mean $\mathrm{BV} / \mathrm{TV}$ were reconstructed for each group. The stress group exhibited significant trabecular bone loss compared with the control group. Trabecular volume was higher in the stress/chewing group than in the stress group.
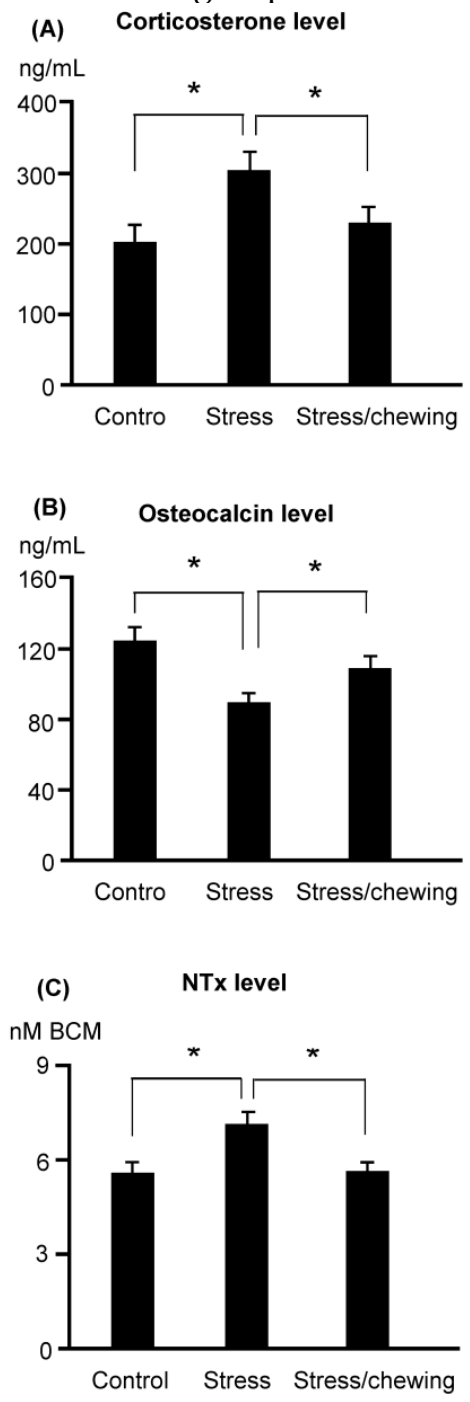

Figure 1. The serum biochemical parameters. (A) Corticosterone, (B) Osteocalcin, and (C) N-telopeptide of type collagen (NTx) levels in the control, stress and stress with chewing mice. All data represent as mean $\pm S D$. $* P<0.05$. BCE: Bone Collagen Equivalents.

Microstructural parameters of the distal femoral trabecular bone are shown in Fig. 4. The pattern of changes in the microstructural properties of the distal femoral trabecular bone was similar to those of vertebral trabecular bone. BV/TV and Tb.Th were significantly lower, and Tb.Sp was significantly higher in the stress group compared with the control group. $\mathrm{BV} / \mathrm{TV}$ was significantly increased, and Tb.Sp was significantly decreased in the stress/chewing group compared with the stress group. Tb.N and Tb.Th tended to be higher in the stress/chewing group as well. 

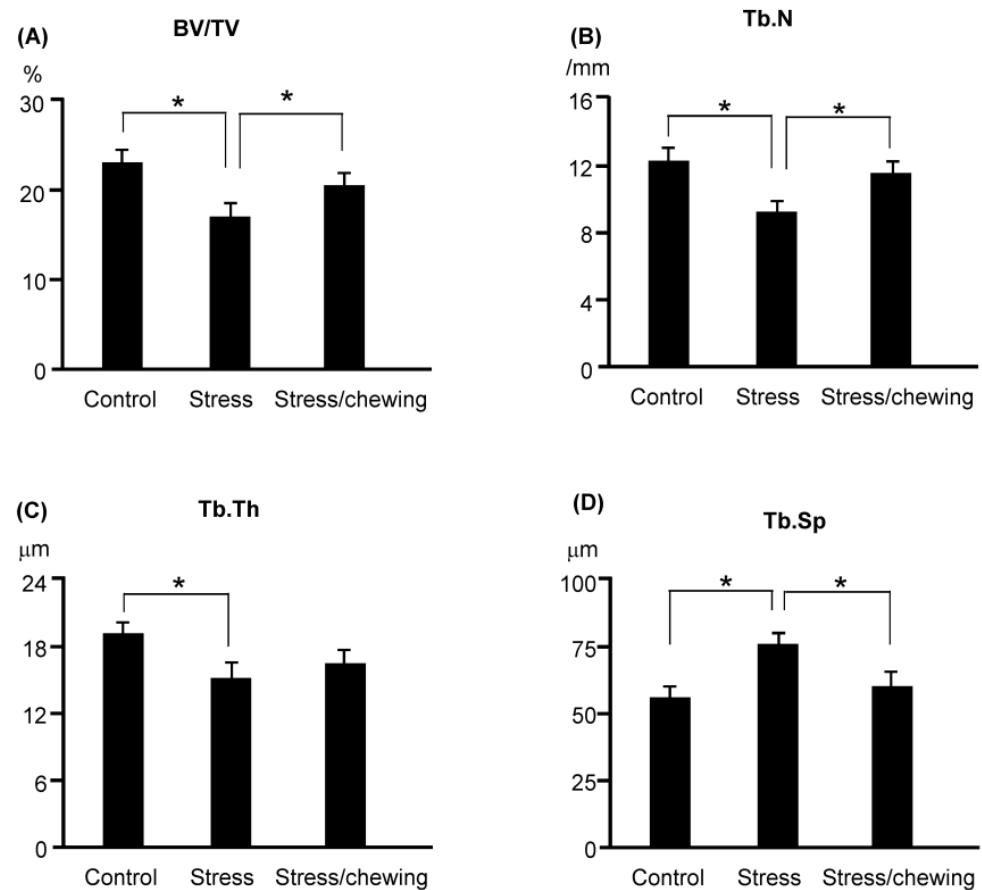

Figure 2. The microstructural parameters of the vertebral trabecular bone. (A) Bone volume fraction (BV/TV), (B) Trabecular number (Tb.N), C Trabecular thickness (Tb.Th), and (D) Trabecular separation (Tb.Sp) in the control, stress, and stress with chewing mice. All data represent as mean \pm SD. $* P<0.05$.

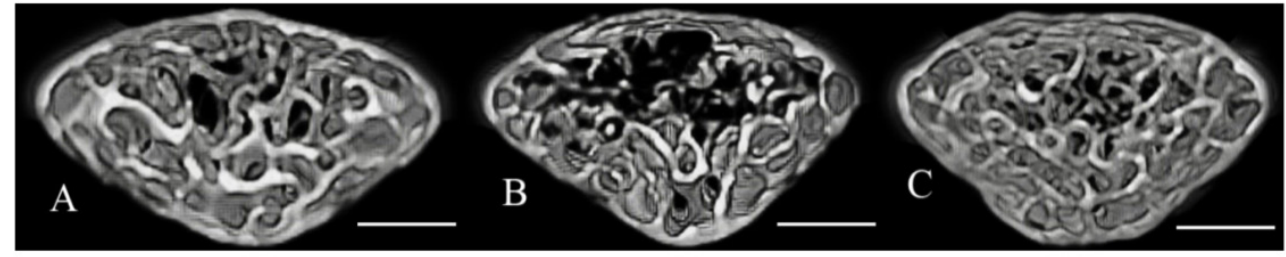

Figure 3. Three-dimensional micro-CT images representative the fourth lumbar vertebral body in the control (A), stress (B), and stress with chewing $(\mathbf{C})$ mice. Images of the sample with the BV/TV that was closest to the mean BV/TV were presented in each group. Scale bar: $0.3 \mathrm{~mm}$.
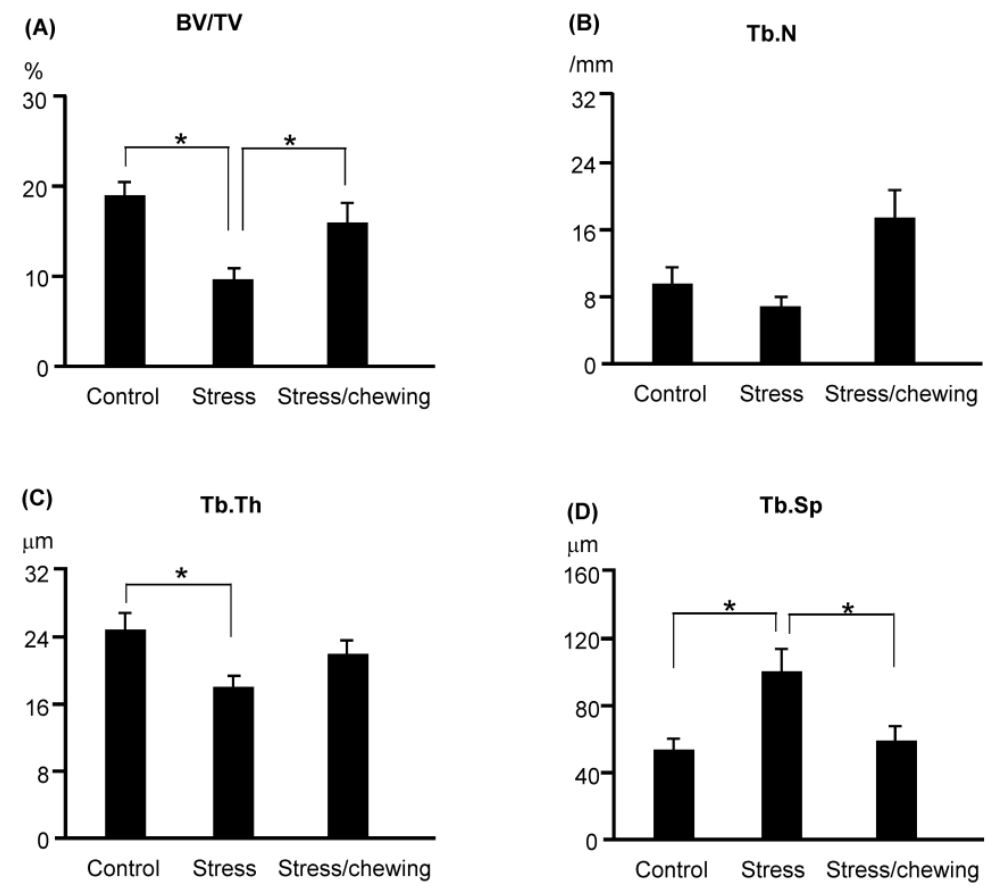

Figure 4. The microstructural parameters of the distal femoral metaphyseal trabecular bone. (A) Bone volume fraction (BV/TV), (B) Trabecular number (Tb.N), (C) Trabecular thickness (Tb.Th), and (D) Trabecular separation (Tb.SP) in the control, stress, and stress with chewing mice. All data represent as mean \pm SD. *P $<0.05$. 
The BFR/BS in the stress group was significantly lower than that in the control group. BFR/BS was significantly higher in the stress/chewing group compared with the stress group (Fig. 5). N.Oc/BS and Oc.S/BS were significantly higher in the stress group compared with the control group (Fig. 5). Active chewing while under chronic stress suppressed the decrease in BFR/BS and suppressed the increases in N.Oc/BS and Oc.S/BS (Fig. 5).
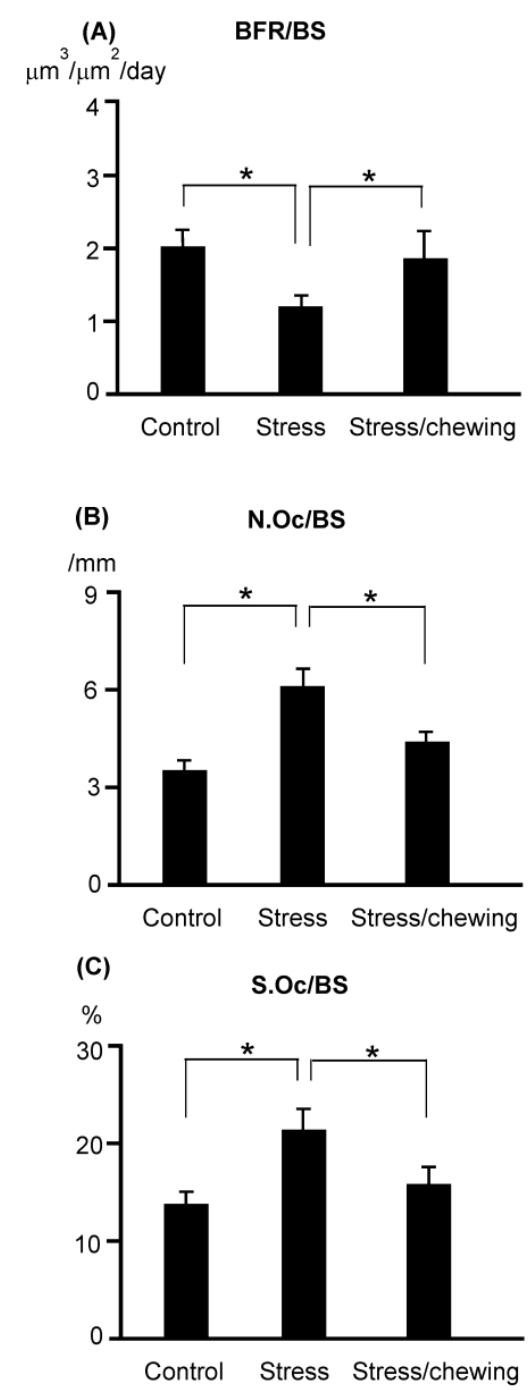

Figure 5. The bone remodeling parameters in the control, stress, and stress with chewing mice. (A) The bone formation rate (BFR/BS), (B) The osteoclast number (N.Oc/BS), and (C) The osteoclast surface (Oc.S/BS). All data represent as mean \pm SD. $* P<0.05$.

\section{Discussion}

The results of the present study were consistent with findings from controlled studies in both humans and experimental animals suggest that exposure to various chronic stressful stimuli inhibits osteocalcin level, decreases osteoblast bone formation, and leads to bone loss [8-10]. In B6 mice, serum osteocalcin lev- els were significantly decreased after exposure to chronic stress for 4 weeks. Chronic stress induced a significant reduction of trabecular bone mass both in the distal femur and vertebral body. Based on histomorphometric measurements, exposure to chronic stress led to a decrease in BV/TV, Tb.N, and Tb.Th, and an increase in Tb.Sp. Chronic stress exposure reduces BV/TV in the distal femur by its effects to decrease $\mathrm{Tb}$.Th and increase Tb.Sp, whereas it leads to reduction in $\mathrm{BV} / \mathrm{TV}$ in the vertebra by its effects to decrease both Tb.N and Tb.Th, and increase Tb.Sp. These effects of stress on trabecular bone loss are consistent with those observed in osteoporotic patients [28, 33, 34]. Bone remodeling, in which the resorption phase is followed by the reformation phase, occurs continuously and is strictly regulated by various factors. Exposure to various chronic stressors, such as restraint, immobilization, forced swimming, noise, crowding, and social stress leads to sustained increases in serum corticosterone levels in rodents [9, 35]. Consistent with this, adrenalectomy abolishes chronic stress-induced behavioral depression and bone loss, supporting the involvement of glucocorticoids in these effects [9]. Increased apoptosis of osteoblasts and osteocytes along with an increased life span of osteoclasts leads to decreased bone mass as well as decreased bone strength [7]. Several recent investigations demonstrated that active mastication inhibits some stress-induced effects in rodents, such as increases in noradrenaline turnover in the rat hypothalamus, limbic regions, and amygdala, and impaired spatial memory resulting from glucocorticoid receptor expression in the hippocampus [21, 22]. Active chewing also attenuates the stress-induced increase in serum corticosterone levels $[18,20]$, rescues stress-impaired hippocampal functions, and improves stress-induced spatial memory [12, 18, 20]. Active mastication also attenuates sympathetic hyperactivity during stress and prevents post-stress arrhythmias [20]. The findings of the present study indicated that active mastication significantly improved bone loss and bone density loss induced by exposure to chronic stress.

In conclusion, the present study demonstrated that chronic mild psychologic stress activates the HPA axis, which leads to increased serum corticosterone, and triggers bone loss by inhibiting osteoblast bone formation and stimulating osteoclast bone resorption. Active mastication suppresses the neuroendocrine stress response to activation of the HPA axis, thereby inhibiting increases in serum corticosterone, attenuates the inhibited bone formation and activated bone resorption, ameliorates trabecular bone loss and bone density loss induced by chronic stress. These findings demonstrated that active mastication can improve 
chronic stress-dependent bone loss in B6 mice. Therefore, active mastication might be an effective approach for the prevention and/or treatment of chronic stress-related osteoporosis.

\section{Acknowledgements}

This work was supported by a Grant-in-Aid for Scientific Research from the Ministry of Education, Science, and Culture of Japan (26462916). The authors thank Dr. Ken-ichi Tezuka, Department of Tissue and Organ Development, Gifu University Graduate School of Medicine, for providing the micro-CT system used in this study.

\section{Competing Interests}

The authors have declared that no competing interest exists.

\section{References}

1. Marin MF, Lord C, Andrews J, et al. Chronic stress, cognitive functioning and mental health. Neurobiol Learn Mem. 2011; 96: 583-595.

2. Proietti R, Mapelli D, Volpe B, et al. Mental stress and ischemic heart disease: evolving awareness of a complex association. Future Cardiol. 2011; 7: 425-437.

3. Reber SO. Stress and animal models of inflammatory bowel disease-an update on the role of the hypothalamo-pituitary-adrenal axis. Psychoneuroendocrinology. 2012; 37: 1-19.

4. Pang $\mathrm{S}, \mathrm{Wu} \mathrm{H}$, Wang $\mathrm{Q}$, et al. Chronic stress suppresses the expression of cutaneous hypothalamic-pituitary-adrenocortical axis elements and melanogenesis. PLoS One. 2014; 9: e98283.

5. NIH. Osteoporosis prevention, diagnosis, and therapy. NIH Consensus Statement. 2000; 17: 1-45.

6. Pothiwala P, Evans EM, Chapman-Novakofski KM. Ethnic variation in risk for osteoporosis among women: a review of biological and behavioral factors. J Womens Health. 2006; 15: 709-719.

7. Weinstein RS. Clinical practice. Glucocorticoid-induced bone disease. N Engl J Med. 2011; 365: 62-70.

8. Patterson-Buckendahl P, Kvetnansky R, Fukuhara K, et al. Regulation of plasma osteocalcin by corticosterone and norepinephrine during restraint stress. Bone. 1995; 17: 467-472.

9. Yirmiya R, Goshen I, Bajayo A, et al. Depression induces bone loss through stimulation of the sympathetic nervous system. Proc Natl Acad Sci USA. 2006; 103: 16876-16881.

10. Cizza G, Primma S, Csako G. Depression as a risk factor for osteoporosis. Trends Endocrinol Metab. 2009; 20: 367-373.

11. Karsenty G, Ferron M. The contribution of bone to whole-organism physiology. Nature. 2012; 481: 314-320.

12. Weinstein RS, Jilka RL, Parfitt AM, Manolagas SC. Inhibition of osteoblastogenesis and promotion of apoptosis of osteoblasts and osteocytes by glucocorticoids. Potential mechanisms of their deleterious effects on bone. J Clin Invest. 1998; 102: 274-282.

13. Henneicke H, Gasparini SJ, Brennan-Speranza TC, et al. Glucocorticoids and bone: local effects and systemic implications. Trends Endocrinol Metab. 2014; 25: 197-211.

14. Onozuka M, Fujita M, Watanabe K, et al. Age-related changes in brain regional activity during chewing: a functional magnetic resonance imaging study. J Dent Res. 2003; 82: 657-660.

15. Ono Y, Kataoka T, Miyake S, et al. Chewing ameliorates stress-induced suppression of hippocampal long-term potentiation. Neuroscience. 2008; 154: 1352-1359.

16. Ono Y, Yamamoto T, Kubo KY, Onozuka M. Occlusion and brain function: mastication as a prevention of cognitive dysfunction. J Oral Rehabil. 2010; 37: 624-640.

17. Hirano $\mathrm{Y}$, Obata $\mathrm{T}$, Takahashi $\mathrm{H}$, et al. Effects of chewing on cognitive processing speed. Brain Cogn. 2013; 81: 376-381.

18. Kubo KY, Sasaguri K, Ono Y, et al. Chewing under restrain stress inhibits the stress-induced suppression of cell birth in the dentate gyrus of aged SAMP8 mice. Neurosci Lett. 2009; 466: 109-113.

19. Ono Y, Kataoka T, Miyake S, et al. Chewing rescues stress-suppressed hippocampal long-term potentiation via activation of histamine $\mathrm{H} 1$ receptor. Neurosci Res. 2009; 64: 385-390.

20. Koizumi S, Minamisawa S, Sasaguri K, et al. Chewing reduced sympathetic nervous response to stress and prevents poststress arrhythmias in rats. Am J Physiol Heart Circ Physiol. 2011; 301: H1551-H1558.
21. Miyake S, Wada-Takahashi S, Honda H, et al. Stress and chewing affect blood flow and oxygen levels in the rat brain. Arch Oral Biol. 2012; 57: 1491-1497.

22. Miyake S, Yoshikawa G, Yamada K, et al. Chewing ameliorates stress-induced suppression of spatial memory by increasing glucocorticoid receptor expression in the hippocampus. Brain Res. 2012; 1446: 34-39.

23. Furuzawa $M$, Chen $H$, Fujiwara $S$, et al. Chewing ameliorates chronic stress-induced bone loss in senescence-accelerated mouse (SAMP8), a murine model of senile osteoporosis. Exp Gerontol. 2014; 55: 12-18.

24. Squire M, Donahue LR, Rubin C, Judex S. Genetic variations that regulate bone morphology in the male mouse skeleton do not define its susceptibility to mechanical unloading. Bone. 2004; 35: 1353-1360.

25. Wergedal JE, Sheng MH, Ackert-Bicknell CL, et al. Genetic variation in femur extrinsic strength in 29 different inbred strains of mice is dependent on variations in femur cross-sectional geometry and bone density. Bone. 2005; 36: 111-122.

26. Glatt V, Canalis E, Stadmeyer L, Bouxsein ML. Age-related changes in trabecular architecture differ in female and male C57BL/6J mice. J Bone Miner Res. 2007; 22: 1197-1207.

27. Uchida S, Hara K, Kobayashi A, et al. Epigenetic status of Gdnf in the ventral striatum determines susceptibility and adaptation to daily stressful events. Neuron. 2011; 69: 359-372.

28. Chen H, Zhou X, Washimi Y, Shoumura S. Three-dimensional microstructure of the bone in a hamster model of senile osteoporosis. Bone. 2008; 43: 494-500.

29. Chen H, Washimi Y, Kubo KY, Onozuka M. Gender-related changes in three-dimensional microstructure of trabecular bone at the human proximal tibia with aging. Histol Histopathol. 2011; 26: 563-570.

30. Chen H, Kubo KY. Segmental variations in trabecular bone density and microstructure of the spine in senescence-accelerated mouse (SAMP6): a murine model for senile osteoporosis. Exp Gerontol. 2012; 47: 317-322

31. Fen JQ, Zhang J, Dallas SL, et al. Dentin matrix protein 1, a target molecule for Cbfa1 in bone, is a unique bone marker gene. J Bone Miner Res. 2002; 17: 1822-1831.

32. Egan KP, Brennan TA, Pignolo RJ. Bone histomorphometry using free and commonly available software. Histopathology. 2012; 61: 1168-1173.

33. Riggs BL, Parfitt AM. (2005) Drugs used to treat osteoporosis: the critical need for a uniform nomenclature based on their action on bone remodeling. J Bone Miner Res. 2005; 20: 177-184.

34. Chen H, Zhou X, Shoumura S, Emura S, Bunai Y. Age- and gender-dependent changes in three-dimensional microstructure of cortical and trabecular bone at the human femoral neck. Osteoporos Int. 2010; 21: 627-636.

35. Konkle AT, Baker SL, Kentner AC, et al. Evaluation of the effects of chronic mild stressors on hedonic and physiological responses: sex and strain compared. Brain Res. 2003; 992: 227-238 